\author{
Vittorio Govoni \\ Enrico Granieri \\ Maria Rosaria Tola \\ Ilaria Casetta \\ Patrizia Ruppi \\ Luca Vaghi
}

\section{The frequency of clinical variants of Guillain-Barré syndrome in Ferrara, Italy}

Received: 2 June 1998

Received in revised form: 29 October 1998

Accepted: 18 May 1999
V. Govoni (西) · E. Granieri · M. R. Tola

I. Casetta · P. Ruppi - L. Vaghi

Sezione di Clinica Neurologica,

Università degli Studi di Ferrara,

Corso della Giovecca 203,

I-44100 Ferrara, Italy

Tel.: +39-03-532236304,

Fax: +39-03-532205525

\begin{abstract}
As the available diagnostic criteria (National Institute of Neurological and Communicative Disorders and Stroke, NINCDS) for Guillain-Barré syndrome (GBS) do not permit inclusion of clinical variants (CV) of GBS, there are few data on their occurrence and few reports of the overall incidence of the disease. A population-based study in the local health district of Ferrara, Italy in 1981-1993 selected cases fulfilling both NINCDS criteria (NINCDS GBS cases) and CV. The incidence of CV was 0.35 per 100,000 personyears (95\% CI: 0.15-0.68), 0.32 when age-adjusted to the Italian population. No difference was found between CV and NINCDS GBS for male/female ratio, mean age at onset, elevated CSF protein content, seasonal pattern, or mean time delay from first neurological symptom to maximal severity. A higher frequency of antecedent infections for CV and more frequent serious disease at
\end{abstract}

the nadir time for NINCDS GBS were found. A complete recovery was more frequent for $\mathrm{CV}$ than NINCDS GBS, but no difference was found regarding good outcome (defined by a satisfactory recovery and resumption of normal functional life). Since most findings were similar for NINCDS GBS and CV cases, they may have similar underlying pathological mechanisms. When diagnostic criteria for GBS include CV, the overall disease incidence in the Ferrara district increases from 1.87 to 2.21 cases per 100,000 personyears (the contribution of $\mathrm{CV}$ to the overall incidence of GBS is $15.7 \%$ ). The currently available diagnostic criteria for GBS, although useful for field studies, may be too restrictive as they can entail the loss of about $15 \%$ of cases.

Key words Guillain-Barré syndrome Clinical variants . Frequency

\section{Introduction}

The clinical polymorphism of Guillain-Barré syndrome (GBS), an acute inflammatory demyelinating polyradiculoneuropathy of unestablished etiology, is remarkable, and its diagnostic limits remain uncertain $[4,10,20]$. The present diagnostic criteria have been established by an ad hoc committee of the National Institute of Neurological and Communicative Disorders and Stroke (NINCDS) [5]. Using NINCDS criteria most population-based studies show an annual incidence in the range of $1-2$ per 100,000 population [2]. NINCDS criteria have proven useful for field studies but have been criticized as being overly restrictive $[4,17]$ as they exclude some varieties of GBS [14]. Clinical variants (CV) of GBS not fulfilling NINCDS criteria have been described as Miller-Fisher syndrome (MFS), polyneuritis cranialis, sensory form, acute pandysautonomia, and chronic inflammatory demyelinating polyneuropathy $[6,8]$. Regional forms (pharyngeal-cervical-brachial variant and paraparetic variant) and a pure motor variant have also been described, but since these satisfy 
NINCDS criteria, they have probably been selected by incidence studies in which NINCDS criteria for GBS were used. As NINCDS criteria do not include CV, there are few data on their occurrence and few reports on the overall incidence of the disease. CV may be relevant in defining the limits of this symptom complex and the relative weight of the typical syndrome compared to its variants. However, there is no overall consensus as to whether GBS fulfilling NINCDS criteria and CV are variants of the same underlying immune disorder, or whether they represent distinct clinical entities. CV have been described mainly from clinical series, which may be subject to selection bias. A population-based study on GBS in the local health district (LHD) of Ferrara, Italy, included both cases fulfilling NINCDS criteria and CV. An average annual incidence of 1.87 per 100,000 population for GBS cases fulfilling NINCDS criteria was estimated [9]. The present paper reports the findings for CV.

\section{Material and methods}

The study population were residents of Ferrara LHD, a well-defined population of northern Italy, in the years from 1981 to 1993 $(2,304,100$ person-years). The methodological approach has been reported elsewhere [9]. In Ferrara LHD there are two general hospitals (in the towns of Ferrara and Bondeno). The two neurological wards of Ferrara LHD are located in Ferrara Hospital (the Neurological Clinic of Ferrara University and another neurological ward). The Bondeno Hospital has a consultant neurologist. No neurological wards exist in the other four LHDs of the Province of Ferrara, and there are no private neurological clinics in the study area. The three private hospitals in Ferrara LHD lack a neurological ward, treat only medical and surgical problems, and are served by the Ferrara Hospital for neurological problems. The Center for Motor Rehabilitation is located in Ferrara. The only Clinical Neurophysiology Service and Neurochemistry Laboratory in the Province of Ferrara are located in the Neurological Clinic of Ferrara Hospital. These have provided consultation to the entire Province of Ferrara since the mid-1970s. All neurological patients in the Province of Ferrara have been referred to Ferrara Hospital since the 1970s. As neurological services in the Province of Ferrara are concentrated in the city of Ferrara (and neurological patients in the Province of Ferrara are referred to Ferrara Hospital), Ferrara LHD is suitable for neuroepidemiological research. The study has been reviewed by the ethics committee of Ferrara Hospital.

Patients were eligible if they were resident within Ferrara LHD (nonresident cases were excluded). The NINCDS diagnostic criteria for GBS were used [5]. Patients fulfilling NINCDS criteria were classified as NINCDS GBS cases. A monophasic illness fulfilling NINCDS criteria with a progressive phase between 4 and 8 weeks, i.e. subacute GBS [10], was considered as a NINCDS GBS case. This is in agreement with NINCDS criteria which state include GBS cases (less than 10\%) with a progression beyond 4 weeks $[5,6]$. Patients with electrophysiological findings consistent with an axonal neuropathy otherwise fulfilling NINCDS criteria were considered as NINCDS GBS cases and not as a CV of GBS [10]. In accordance with Asbury [6] we considered as CV of GBS the MFS (acute ophthalmoplegia, ataxia, and generalized tendon areflexia in the absence of relevant limb weakness), the sensory form (sensory loss and tendon areflexia without relevant limb weakness), polyneuritis cranialis, acute pandysautonomia, and other CV (other acute monophasic peripheral nervous system diseases with albuminocytological dissociation in the cerebrospinal fluid not fulfilling NINCDS criteria). To compare our results with those of other epidemiological studies [7, 11, 16, 19] chronic inflammatory demyelinating polyradiculoneuropathy was not considered a CV of GBS. This is in agreement with other authors who suggested considering chronic inflammatory demyelinating polyradiculoneuropathy (whose diagnostic criteria require, as mandatory, a progressive phase of at least 2 months) [11] separately from GBS and not as a clinical variant of it $[4,10,12]$. To compare our findings about the incidence of NINCDS GBS and its CV with those of other epidemiological studies we excluded (if encountered) cases of human immunodeficiency virus (HIV) associated neuropathy of the GBS type [11, 19]. HIV-associated neuropathy of the GBS type most often occurs at the time of seroconversion, but it seems to be a rare event [12]. However, none of the cases of HIV-associated neuropathy found in the study satisfied NINCDS criteria for GBS (and would therefore not have been selected in any event). Cases were accepted as $\mathrm{CV}$ when primary causes of neuropathy (such as diabetes and intoxications) had been ruled out.

Incident cases of GBS in Ferrara LHD were identified prospectively because acute or subacute neurological patients in the Province of Ferrara are routinely referred to the two neurological wards of Ferrara Hospital. For completeness of case ascertainment an intensive restrospective survey was performed of all possible sources of GBS cases for the entire study period. A complete enumeration approach was used. Sources of case material were the medical records of the two neurological wards of Ferrara Hospital, of the Center for Motor Rehabilitation of Ferrara LHD, of the intensive care, pediatric, internal medicine, and geriatric wards of the two general hospitals of Ferrara LHD, and the files of the Clinical Neurophysiology Service and the Neurochemistry Laboratory of Ferrara Hospital.

All practicing neurologists in Ferrara LHD and in the other four LHDs of the Province of Ferrara were interviewed. The general practitioners of the Province were contacted through announcements in the official publication of the Physician's Chamber in the Province (which is sent periodically to all physicians in the Province). The head physicians of intensive care, pediatric, internal medicine, and geriatric wards of the hospitals of the other four LHDs in the Province were questioned by letter. To check completeness of case ascertainment we reviewed the discharge diagnoses of Ferrara LHD, codified according to the International Classification of Diseases, 9th revision. All discharge diagnoses (primary or secondary diagnoses) were taken that were coded 357 (inflammatory and toxic neuropathy), 356.4 (progressive idiopathic polyneuropaty), 356.8 (others), 356.9 (unspecified). The clinical files of the abstracted discharge diagnoses were obtained from each hospital ward. The research plan of the study was approved by the Chief Executive and the Management of Ferrara LHD, and the authors were authorized to review the clinical files of the cases and the hospital discharge data. The abstracted clinical files from all sources corresponding to possible cases were examined exclusively by the team. The data on each possible case were reviewed jointly by the senior authors. The diagnosis of NINCDS GBS and CV was made by collective discussion to achieve unanimity on each case.

Patients were followed up for 1-year to determine outcome, and they were graded using a disability scale [15]: grade 0 , healthy (no signs or symptoms due to GBS or CV); grade 1, minor symptoms or signs and able to run; grade 2 , able to walk $5 \mathrm{~m}$ across an open space without assistance, walking frame or stick but unable to run; grade 3 , able to walk $5 \mathrm{~m}$ across an open space with the help of one person and waist level walking frame or sticks; grade 4 , chair-bound or bedbound (unable to walk as in 3); grade 5, requiring mechanical ventilator; grade 6 , death. Outcome was determined according to functional recovery of the patients. A good outcome was defined by a satisfactory recovery and resumption of normal functional life (disability grade 0 or 1 ). Poor outcome was defined by persistent disability or death during admission to hospital (disability grade of 2 or greater). 
The $95 \%$ confidence interval (CI) of rates was computed assuming a Poisson distribution [18]. The crude rates were age-adjusted by the direct method using the Italian population at the 1981 general census as a standard. The $\chi^{2}$ test for linear trend was used when appropriate [3]. Seasonal preponderance was tested by goodness-of-fit $\chi^{2}$ test, assuming a null hypothesis of no seasonal variation. The Student's $t$ test was used for comparing means and the log-likelihood ratio test was used to compare proportions. The $Z$ test was used to compare two rates.

\section{Results}

The study selected 51 patients, 43 of whom fulfilled NINCDS criteria for GBS while the other 8 (who did not satisfy NINCDS criteria) were considered CV. Among the NINCDS GBS cases 3 patients $(6.9 \%)$ showed a progressive phase between 4 and 8 weeks, and another patient $(2.3 \%)$ had electrophysiological findings which were consistent with an axonal neuropathy. No cases of HIV-associated neuropathy fulfilling NINCDS criteria were found. All 8 patients with CV and 40 NINCDS GBS cases (94.1\% of selected patients) were identified in the prospective survey. The retrospective study identified the other 3 NINCDS GBS cases (5.9\%). The review of International Classification of Diseases (9th revision) defined discharge diagnoses codes in Ferrara LHD supplied no further cases. Based on 43 cases the incidence for NINCDS GBS was 1.87 per 100,000 person-years $(95 \%$ CI: 1.35-2.52), 1.66 when age-adjusted.

The eight CV cases were: three MFS, four polyneuritis cranialis, and one sensory form. No cases consistent with acute pandysautonomia were found. All three MFS had elevated CSF protein content. The four polyneuritis cranialis (one patient with acute facial diplegia and perioral paresthesias; one patient with acute facial diplegia and generalized tendon hyporeflexia; one patient with facial diplegia, reduced tendon reflexes, distal hypopallesthesia, and paresthesias in the limbs; one patient with dysarthria and dysphagia showing palatopharyngeal palsy, slight asymmetric bifacial weakness predominating on the right, and generalized tendon hyporeflexia) showed increased CSF protein concentration and complete recovery. Based upon a pure sensory syndrome with an acute monophasic clinical course, complete recovery, elevated CSF protein content, and electrophysiological evidence of demyelinative process of peripheral nervous system (severe sensory nerve conduction abnormalities) the last $\mathrm{CV}$ was considered a sensory form (one patient presenting with paresthesias in the feet and hands, rapid symmetrical sensory loss in the limbs mainly involving vibration and joint position, generalized tendon areflexia, no motor involvement) [6, 13]. Based on these eight cases the $\mathrm{CV}$ incidence rate was 0.35 per 100,000 person-years (95\% CI: $0.15-0.68$ ), 0.32 when age-adjusted. CV and NINCDS GBS in Ferrara LHD showed no significant difference in male/female ratio (1.67 and 1.50) or mean age at onset (48.0 \pm 17.3 and $52.9 \pm 18.1$ years $)$.
Table 1 Age-specific incidence rate of NINCDS GBS and CV cases per 100,000 person-years in Ferrara LHD, Italy, 1981-1993

\begin{tabular}{lcccccc}
\hline & & \multicolumn{2}{c}{ NINCDS GBS } & & \multicolumn{2}{l}{ CV } \\
Age (years) & Person-years & $n$ & Rate* & & $n$ & Rate \\
\hline $0-29$ & 788,002 & 7 & 0.89 & & 3 & 0.38 \\
$30-59$ & 993,067 & 16 & 1.61 & & 3 & 0.30 \\
$60+$ & 523,031 & 20 & 3.82 & & 2 & 0.38 \\
Total & $2,304,100$ & 43 & 1.87 & & 8 & 0.35 \\
\hline
\end{tabular}

$* P<0.001, \chi^{2}$ test for linear trend $=13.475$

All CV and 40 NINCDS GBS (93.0\%) underwent at least one CSF examination. Considering only CSF examinations performed after the first week of symptoms, an increased CSF protein concentration was found in $100 \%$ of CV and $92.5 \%$ of NINCDS GBS (n.s.). An acute infectious illness within 1 month before disease onset was reported more frequently for $\mathrm{CV}$ than NINCDS GBS $(87.5 \%$ and $46.5 \%$; log-likelihood ratio test $=5.079, P<$ $0.05)$. Antecedent events for NINCDS GBS were upper respiratory infections $(45.0 \%)$, gastrointestinal infections (20.0\%), fever of unknown origin $(25.0 \%)$, otitis $(5.0 \%)$, and phlebitis $(5.0 \%)$ while they were upper respiratory infections $(50.0 \%)$, gastrointestinal infections $(12.5 \%)$ and fever of unknown origin $(25.0 \%)$ for CV. Both CV (winter $37.5 \%$, spring $12.5 \%$, summer $25.0 \%$, autumn $25.0 \%$ ) and NINCDS GBS (winter $37.5 \%$, spring $15.0 \%$, summer $32.5 \%$, autumn $15.0 \%$ ) showed a higher incidence in winter but no significant seasonal clustering. An increase in incidence with age was found for NINCDS GBS $\left(\chi^{2}\right.$ test for linear trend $=13.475, P<0.001$ ) but not for CV (Table 1). CV showed an increase in incidence over the study period (three following time intervals of the study period: 1981-1984, 1985-1988, 1989-1993, incidence rates 0.14, 0.28 , and 0.58 per 100,000 person-years, $\chi^{2}$ test for linear trend $=7.347, P<0.01$ ) as previously reported for NINCDS GBS (three following time intervals: 19811984, 1985-1988, 1989-1993, incidence rates 1.09, 1.83, and 2.55 per 100,000 person-years, $\chi^{2}$ test for linear trend $=$ 4.491, $P<0.05)$ [9].

NINCDS GBS incidence was higher in urban than rural areas of Ferrara LHD (2.22 and 1.10 per 100,000 person-years, $Z=2.082, P<0.05$ ) [9] while a nonsignificant urban-rural difference was found for CV (0.38 and 0.27 per 100,000 person-years). The mean time delay from first neurological symptom to nadir (maximal severity) was $12.4 \pm 11.3$ days for NINCDS GBS and $13.1 \pm 10.5$ days for CV (n.s.). At the time of their nadir 27 NINCDS GBS patients $(62.8 \%)$ reached grades 3 and 4 of the disability scale, $10(23.3 \%)$ grades 1 and 2, while $6(14.0 \%)$ needed ventilatory assistance (grade 5). Among CV grade 1 accounted for the 4 polyneuritis cranialis $(50.0 \%)$, grade 2 for 1 MFS and the sensory form (25.0\%), and grade 3 for the other 2 MFS (25.0\%) because of a relevant ataxic gait. 
Therefore a mild disease (grades 1 and 2) was more common among CV (75.0\%) than NINCDS GBS (log-likelihood ratio test $=7.809, P<0.01)$. Four patients $(9.3 \%)$ with NINCDS GBS and none with CV died during the stay in hospital (n.s.). The reported causes of death were acute cardiorespiratory failure in two patients, respiratory failure and pulmonary embolism. One year after onset all $\mathrm{CV}$ and 26 NINCDS GBS patients $(60.5 \%)$ had recovered $(\log$-likelihood ratio test $=7.223, P<0.01)$. A good outcome (disability grade 0 or 1 ) was found in $100.0 \%$ of $\mathrm{CV}$ and $79.1 \%$ of NINCDS GBS (n.s.). The overall recovery was $66.7 \%$ while a good outcome was found in 42 of 51 cases $(82.4 \%)$.

\section{Discussion}

The CV incidence in Ferrara LHD was 0.35 per 100,000 person-years. If diagnostic criteria for GBS include CV, the overall disease incidence in Ferrara LHD increases from 1.87 to 2.21 per 100,000 person-years $(95 \% \mathrm{CI}$ : 1.65-2.89), and the contribution of CV to the overall incidence of GBS becomes $15.7 \%$. For CV defined diagnostic criteria have not been established, and their frequency has usually been estimated in clinical series which could be subject to selection bias. Arnason and Soliven] reported that CV constitute up to $15 \%$ of GBS [4], which is similar to the figure found in Ferrara LHD. Clinical variants of GBS occurred in $18 \%$ of the patients in a prospective series [15] and represent $11 \%$ and $13 \%$ of cases in population-based studies [7, 17]. The reported frequency of MFS ranges from $2 \%$ to $7 \%[7,14,15,17]$. The MFS frequency found in Ferrara LHD was 6\%, which is in the range of the previously reported estimations.

The present findings derive from a population-based study in the well-defined population of Ferrara LHD, and selection bias is therefore unlikely. Moreover, case ascertainment (through a prospective survey followed by an intensive retrospective study of all possible sources of case material) seems to be complete. In fact, in spite of the population size, our findings (similar to the previously reported data) seem to be reliable. Reliability is also supported by the high percentage of patients observed with mild disease at nadir. However, it must be emphasized that the present findings are based on few observations. No difference was found between NINCDS GBS and CV in male/female ratio, mean age at onset, elevated CSF protein content, seasonal pattern, or mean time delay from first neurological symptom to maximal severity. Moreover, as has been reported for NINCDS GBS [9], CV showed a slight increase in incidence over the study period. An increase in incidence with age and a higher incidence in urban zone, reported for NINCDS GBS [9], were not found for CV. CV differed from NINCDS GBS since they showed a higher frequency of acute infections before disease onset. Moreover, a mild disease at the nadir time was more frequent in CV than in NINCDS GBS and CV recovered more frequently than NINCDS GBS. However, no difference between CV and NINCDS GBS was found for good outcome (disability grade 0 or 1 ). Therefore most of the epidemiological findings were similar for NINCDS GBS and CV cases which occurred in Ferrara LHD. The main differences were a higher frequency of antecedent infections for $\mathrm{CV}$ and a more frequent serious disease at the nadir time for NINCDS GBS.

It is possible that NINCDS GBS and CV are variants of the same underlying immune disorder rather than representing distinct clinical entities (the underlying pathogenetic mechanisms may produce patterns of neurological deficit which differ from NINCDS GBS, so that other acute neuropathic syndromes may be due to the same pathological process as GBS). The present findings could support this notion. However, before any conclusion can be drawn from such findings, it should be further confirmed in larger series. Moreover, it is necessary to emphasize that the inclusion of $\mathrm{CV}$ could raise some problems. In fact, when performing therapeutic trials it is crucial to deal with homogeneous groups of patients. Therefore the inclusion of $\mathrm{CV}$ would make the result more difficult to interpret. In conclusion, in the present population-based study CV accounted for $15.7 \%$ of all cases of GBS and showed a good prognosis. Thus, the current diagnostic criteria for GBS, although useful for field studies, may be too restrictive as they can cause the loss of about $15 \%$ of cases.

Acknowledgements This work was supported by the Italian Ministry of University and Scientific and Technological Research $(60 \%)$. We thank the Chief Executive and the Management of Ferrara LHD and Dr. Cristina Polesinanti and Franco Guandalini from the Electronic Data Processing Center of Ferrara Local Health District.

\section{References}

1. Ad Hoc Subcommittee of the American Academy of Neurology AIDS Task Force (1991) Research criteria for the diagnosis of chronic inflammatory demyelinating polyradiculoneuropathy (CIDP). Neurology 41:617-618
2. Alter M (1990) The epidemiology of Guillain-Barré syndrome. Ann Neurol 27 [Suppl]:7-12

3. Armitage P (1955) Tests for linear trends in proportions and frequencies. Biometrics 11:375-386
4. Arnason BGW, Soliven B (1993)

Acute inflammatory demyelinating polyradiculoneuropathy. In: Dyck PJ, Thomas PK, Griffin JW, Low PA, Poduslo JF (eds) Peripheral Neuropathy. Saunders, Philadelphia, pp 1437-1497 
5. Asbury AK, Arnason BG, Karp HR, McFarlin DE (1978) Criteria for diagnosis of Guillain-Barré syndrome. Ann Neurol 3:565-567

6. Asbury AK (1981) Diagnostic considerations in Guillain-Barré syndrome. Ann Neurol 9 [Suppl]:1-5

7. Beghi E, Bogliun $\mathrm{G}$ for the Italian GBS Study Group (1996) The GuillainBarré syndrome (GBS). Implementation of a register of the disease on a nationwide basis. ltal J Neurol Sci 17: 355-361

8. Dowling PC, Blumberg BM, Cook SD (1987) Guillain-Barré syndrome. In: Vinken PJ, Bruyn GW, Klawans HL, Matthews WB (eds) Handbook of clinical neurology. Neuropathies, vol 51. Elsevier, Amsterdam, pp 239-262

9. Govoni V, Granieri E, Casetta I, Tola MR, Paolino E, Fainardi E, Monetti VC (1996) The incidence of GuillainBarré syndrome in Ferrara, Italy: is the disease really increasing? J Neurol Sci 137:62-68
10. Hughes RAC (1995) The concept and classification of Guillain-Barré syndrome and related disorders. Rev Neurol (Paris) 151:5:291-294

11. Lyu R-K, Tang L-M, Cheng S-Y, Hsu W-C, Chen S-T (1997) Guillain-Barré syndrome in Taiwan: a clinical study of 167 patients. J Neurol Neurosurg Psychiatry 63:494-500

12. Martyn CN, Hughes RAC (1997) Epidemiology of peripheral neuropathy. J Neurol Neurosurg Psychiatry 62: 310-318

13. Miralles F, Montero J, Rene R, Matos JAM (1992) Pure sensory GuillainBarré syndrome. J Neurol Neurosurg Psychiatry 55:411-412

14. Poser CM (1981) Criteria for the diagnosis of the Guillain-Barré syndrome: a critique of the NINCDS guidelines. J Neurol Sci 52:191-199

15. Rees JH, Soudain SE, Gregson NA, Hughes RAC (1995) Campylobacter jejuni infection and Guillain-Barré syndrome. N Engl J Med 333:1374-1380
16. Rees JH, Thompson RD, Smeeton NC, Hughes RAC (1998) Epidemiological study of Guillain-Barré syndrome in south east England. J Neurol Neurosurg Psychiatry 64:74-77

17. Ropper AH, Wijdicks EFM, Truax BT (1991) Guillain-Barré syndrome. Davis, Philadephia

18. Schoenberg BS (1983) Calculating confidence intervals for rates and ratios: simplified method utilizing tabular values based on the Poisson distribution. Neuroepidemiology 2:257-265

19. Sedano MJ, Calleja J, Canga E, Berciano J (1994) Guillain-Barré syndrome in Cantabria, Spain. An epidemiological and clinical study. Acta Neurol Scand 89:287-292

20. Thomas PK (1992) The Guillain-Barré syndrome: no longer a simple concept. J Neurol 239:361-362 